\title{
TOTAL INCREMENTAL ITERATIVE FORCE RECOVERY METHOD AND THE APPLICATION IN PLASTIC HINGE ANALYSIS OF STEEL FRAMES
}

\author{
Fawu Wang ${ }^{1, *}$ and Yaopeng Liu $^{2}$ \\ ${ }^{l}$ Department of Civil Engineering, Nanjing University of Aeronautics and Astronautics, Yudao Street, Nanjing, China \\ ${ }^{2}$ Department of Civil and Structural Engineering, \\ The Hong Kong Polytechnic University, Hunghom, Kowloon, Hong Kong \\ *(Corresponding author: E-mail: fwwang@nuaa.edu.cn)
}

Received: 15 January 2008; Revised: 15 February 2008; Accepted: 20 October 2008

\begin{abstract}
In this paper a total incremental iterative force recovery method suitable to plastic hinge analysis is proposed and it is found that this incremental iteration force recovery procedure has a convergence rate comparable to total secant method. The incremental force recovery manner makes this method be capable of recovering member internal forces and deformations correctly and conveniently in the existence of plastic hinge. The capability of the proposed total incremental iterative force recovery method to cope with plastic hinge is illustrated by two benchmark examples.
\end{abstract}

Keywords: Nonlinear analysis; steel frames; force recovery procedure; plastic hinge

\section{INTRODUCTION}

In the former paper [1] a new total incremental iterative force recovery method is proposed by the authors, which resembles incremental iterative system solution technique and utilizes both the secant and tangent stiffness. It is found through the numerical examples that this incremental iteration force recovery procedure has a convergence rate comparable to total secant iteration method and remains the incremental force recovery manner. This paper provides a detailed description about the application of this new incremental force recovery method in the plastic hinge analysis of framed structures.

Geometric nonlinearity, material plasticity and semi-rigid connections are the most important nonlinear sources of framed structures. When material plasticity is involved, there are mainly two types of methods: (1) plastic zone method; or (2) plastic hinge method. The plastic zone method discretizes each member into many fiber segments longitudinally and transversely and the plastic hinge method assumes plasticity lumped only at specific location along an element the other part of element remains elastic. Although the plastic zone solution may be considered exact, it is not conducive to daily use in engineering design because it is too computationally intensive. On the other hand the refined plastic hinge method can approximate the effects of distributed plasticity along the element length and hinge gradual formation, and remains relatively efficient and economical[2-5].

In practice the incremental and iterative solution strategy is employed to trace the load vs. deflection path of a structure, which can be divided into three stages: the predictor, corrector and error-checking phases. The corrector or force recovery procedure determines the accuracy of the solution while the predictor affects only the convergence speed and direction of iteration [6]. Different solution schemes such as arc-length method can be used to traverse the limit points and capture the post-buckling analysis behavior of the structures [7]. 
The load increment sizes control technique has to be used to inspect the forming of plastic hinge in plastic hinge analysis, which scales back the increment size to the yield surface so that the element forces of specific cross-section comply 'exactly' with those on the yield surface [8]. This technique works for plastic hinge analysis but is tedious and low efficiency as plastic hinge is member based and may occur frequently for complex practical structures. Although a lower limit can be specified to avoid small step length in the case of frequent occurrence of yield hinges, 'exact' scaling to the yield surface is not always possible $[9,10]$. In spite of rapidly increased computed speed and memory capacity of personal computers, the computational efficiency is crucial for the daily use of nonlinear or advanced analysis which requires extensive computational time and effort involving hundreds of load cases and thousands of iterations, not to say when design optimization is used where many design parameters are changed and optimized continuously [11].

Actually in advanced analysis and design the behavior of the whole structure is drawn more attention than the exact time or load step when specific plastic hinge occurs. For the incremental and iterative solution strategy the change in the stiffness of practical complex structures caused by plastic hinge has little difference with that caused by second order effect. The equilibrium path of a structure can be obtained by incremental and iterative solution strategy if the element behavior is correctly modeled no matter in elastic range and plastic range. As the corrector determines the accuracy of the modeling, it is natural and neat to inspect the forming of plastic hinge and recover the correct internal forces and deformations accordingly in force recovery procedure.

In the following of this paper Section 2 summarizes the popular beam-column elements in co-rotational formulation. A simple elastic-plastic hinge model is adopted and section spring is used to model the plastic hinge for consistency in Section 3. The approach of modifying different force recovery procedure for plastic hinge analysis is detailed In Section 4 . The capability of the proposed total incremental iteration force recovery method to cope with plastic hinge is illustrated by two classic numerical examples.

\section{BEAM-COLUMN ELEMENT}

Co-rotational formulation is suitable for the geometrically nonlinear analysis of framed structure as the reference frame of every element can be easily determined by the positions of two nodes of the element. In co-rotational method the displacement $\mathrm{u}$ of element can be decomposed into two parts: the rigid body displacements $\mathrm{u}_{r}$ and the natural deformations $\mathrm{u}_{l}$. The effect of the rigid body displacements $\mathrm{u}_{r}$ is to rotate the initial nodal forces from the last configuration to the present configuration without generation of additional internal forces. The separation of the rigid body displacements from the element deformation makes the formulation of beam-column element simplified as the large deflection and rotation effect can be considered in the deformation extraction operation.

The simplest beam-column element is the cubic element which is robust and successful except for the necessity of using several elements for each member to obtain sufficient accuracy [12]. The stability function element [13] and PEP element [14] are capable of dealing with second-order analysis using one element per member in practical application, which simplifies the analysis and design work as there is no difference between the analysis model and the physical model.

In general the secant stiffness relationships of beam-column element can be expressed as

$$
M_{1 n}=\frac{E I_{n}}{L}\left[\left(c_{1 n}+c_{2 n}\right) \theta_{1 n}+\left(c_{1 n}-c_{2 n}\right) \theta_{2 n}\right]
$$




$$
\begin{aligned}
& M_{2 n}=\frac{E I_{n}}{L}\left[\left(c_{1 n}-c_{2 n}\right) \theta_{1 n}+\left(c_{1 n}+c_{2 n}\right) \theta_{2 n}\right] \\
& P=E A\left[\frac{e}{L}+\sum_{n=x, y} b_{1 n}\left(\theta_{1 n}+\theta_{2 n}\right)^{2}+b_{2 n}\left(\theta_{1 n}-\theta_{2 n}\right)^{2}\right] \\
& M_{x}=\frac{G J+\operatorname{Pr}^{2}}{L} \theta_{x}
\end{aligned}
$$

Where $n=x, y$ are two principal directions of member; $e=$ elongation of the element; $f=\left\{M_{1 \mathrm{y}}, M_{2 \mathrm{y}}\right.$, $\left.M_{1 \mathrm{z}}, M_{2 \mathrm{z}}, P, M_{\mathrm{x}}\right\}^{\mathrm{T}}$ is local internal force vector and $u=\left\{\theta_{1 \mathrm{y}}, \theta_{2 \mathrm{y}}, \theta_{1 \mathrm{z}}, \theta_{2 \mathrm{z}}, e, \theta_{\mathrm{x}}\right\}^{\mathrm{T}}$ is local deformation vector; and $E=$ elasticity modulus; $I_{n}=$ moment of inertia; $A=$ section area; $L=$ length of the element; And $c_{1 \mathrm{n}}, c_{2 \mathrm{n}}$ and $b_{1 \mathrm{n}}, b_{2 \mathrm{n}}$ are axial force relating coefficients and has different forms for different types of element and is not repeated here. It should be noted that the last term of Eq. 3 represents the bowing effect which plays important role in the nonlinear interaction of moments and axial force.

The tangent stiffness matrix can be obtained by taking a variation of the secant stiffness with respect to the displacement degrees of freedom and the axial force by which the second-order effect is considered. And the final expression of the incremental stiffness relationship can be written as

$$
\Delta f=\left[k_{e}\right] \Delta u
$$

Where $\Delta f$ and $\Delta u$ are incremental internal force and deformation vector; $\left[k_{\mathrm{e}}\right]$ is element tangent stiffness matrix. The element tangent stiffness matrix in global coordinates and the global tangent stiffness matrix of the whole structure can be transformed and assembled using the standard procedure of co-rotational formulation and the finite element analysis.

\section{ELASTIC-PLASTIC-HINGE MODEL}

As the main point of this paper is not details of the plastic hinge model, the simple elastic-plastic hinge model is selected to illustrate the application of the proposed force recovery method in plastic hinge analysis. In the elastic-plastic hinge model the cross-section behavior is elastic-perfectly plastic and the plasticity is accounted for by zero-length plastic hinges at two ends of each element.

As the cross-section is assumed to be either ideally elastic or fully plastic, the initial yield surface and fully plastic yield surface are superposed which may be represented by AISC LRFD bilinear interaction equations

$$
\begin{array}{ll}
\frac{P}{P_{y}}+\frac{8}{9} \frac{M}{M_{p}}=1.0 & \text { for } \frac{P}{P_{y}} \geq 0.2 \\
\frac{P}{2 P_{y}}+\frac{M}{M_{p}}=1.0 & \text { for } \frac{P}{P_{y}}<0.2
\end{array}
$$


Where $P_{y}=$ the squash load of the cross section; $M_{p}=$ the plastic moment capacity for member under pure bending action; and $P$ and $M=$ the second-order axial force and bending moment at the cross section being considered.

Although the incremental force-displacement relationships can be deduced for different situations such as plastic hinges at one end and both ends, the section spring concept has the merits of simplicity and consistence. The main idea of the section spring model is the same as semi-rigid spring model that a deformed beam-column element with two springs each attached to the element end node, and in fact the two kinds of springs can be combined as springs in series to simulate semi-rigid connection and plastic hinge together $[15,16]$.

The moment- rotation relationship of the section spring can be written as

$$
\begin{aligned}
& M_{1 n}=k_{1 n}^{s}\left(\theta_{1 n}-\theta_{1 n}^{b}\right)=k_{1 n}^{s} \theta_{1 n}^{s} \\
& M_{2 n}=k_{2 n}^{s}\left(\theta_{2 n}-\theta_{2 n}^{b}\right)=k_{2 n}^{s} \theta_{2 n}^{s}
\end{aligned}
$$

And the spring tangent stiffness can be expressed in the incremental form as

$$
\begin{aligned}
& \Delta M_{1 n}={ }^{t} k_{1 n}^{s}\left(\Delta \theta_{1 n}-\Delta \theta_{1 n}^{b}\right)={ }^{t} k_{1 n}^{s} \Delta \theta_{1 n}^{s} \\
& \Delta M_{2 n}={ }^{t} k_{2 n}^{s}\left(\Delta \theta_{2 n}-\Delta \theta_{2 n}^{b}\right)={ }^{t} k_{2 n}^{s} \Delta \theta_{2 n}^{s}
\end{aligned}
$$

The total and incremental rotational stiffness relationships of beam element can be extracted from the secant stiffness relationship in Eqs. 1 to 4 and the tangent stiffness matrix shown in Eq. 5 respectively

$$
\begin{aligned}
& M_{1 n}=\left(k_{11}^{b}\right)_{n} \theta_{1 n}^{b}+\left(k_{12}^{b}\right)_{n} \theta_{2 n}^{b} \\
& M_{2 n}=\left(k_{21}^{b}\right)_{n} \theta_{1 n}^{b}+\left(k_{22}^{b}\right)_{n} \theta_{2 n}^{b} \\
& \Delta M_{1 n}=\left({ }^{t} k_{11}^{b}\right)_{n} \Delta \theta_{1 n}^{b}+\left({ }^{t} k_{12}^{b}\right)_{n} \Delta \theta_{2 n}^{b} \\
& \Delta M_{2 n}=\left({ }^{t} k_{21}^{b}\right)_{n} \Delta \theta_{1 n}^{b}+\left({ }^{t} k_{22}^{b}\right)_{n} \Delta \theta_{2 n}^{b}
\end{aligned}
$$

Through moment equilibrium condition, the additional internal degree-of-freedoms can be eliminated via the static condensation procedure and finally the total and incremental stiffness relationships can be written as

$$
\begin{aligned}
& {\left[\begin{array}{l}
M_{1 n} \\
M_{2 n}
\end{array}\right]=\frac{1}{\beta_{n}^{\prime}}\left[\begin{array}{cc}
k_{1 n}^{s} \beta_{n}^{\prime}-k_{1 n}^{s} k_{1 n}^{s}\left[\left(k_{22}^{b}\right)_{n}+k_{2 n}^{s}\right] & k_{1 n}^{s} k_{2 n}^{s}\left(k_{21}^{b}\right)_{n} \\
k_{1 n}^{s} k_{2 n}^{s}\left(k_{12}^{b}\right)_{n} & k_{2 n}^{s} \beta_{n}^{\prime}-k_{2 n}^{s} k_{2 n}^{s}\left[\left(k_{11}^{b}\right)_{n}+k_{1 n}^{s}\right.
\end{array}\right]\left[\begin{array}{l}
\theta_{1 n} \\
\theta_{2 n}
\end{array}\right]} \\
& \left.\left[\begin{array}{l}
\Delta M_{1 n} \\
\Delta M_{2 n}
\end{array}\right]=\frac{1}{{ }^{t} \beta^{\prime}}\left[\begin{array}{cc}
k_{1 n}^{s} \beta^{\prime}-k_{1 n}^{s} k_{1 n}^{s}\left[\left(k_{22}^{b}\right)_{n}+k_{2 n}^{s}\right] & k_{1 n}^{s} k_{2 n}^{s}\left(k_{21}^{b}\right)_{n} \\
k_{1 n}^{s} k_{2 n}^{s}\left(k_{12}^{b}\right)_{n} & k_{2 n}^{s} \beta_{n}^{\prime}-k_{2 n}^{s} k_{2 n}^{s}\left[\left(k_{11}^{b}\right)_{n}+k_{1 n}^{s}\right.
\end{array}\right]\right]\left[\begin{array}{l}
\Delta \theta_{1 n} \\
\Delta \theta_{2 n}
\end{array}\right]
\end{aligned}
$$


Where

$$
\begin{aligned}
& \beta_{n}^{\prime}=\left[\left(k_{11}^{b}\right)_{n}+k_{1 n}^{s}\right]\left[\left(k_{22}^{b}\right)_{n}+k_{2 n}^{s}\right]-\left(k_{12}^{b} k_{21}^{b}\right)_{n} \\
& { }^{t} \beta_{n}^{\prime}=\left[\left({ }^{t} k_{11}^{b}\right)_{n}+{ }^{t} k_{1 n}^{s}\right]\left[\left({ }^{t} k_{22}^{b}\right)_{n}+{ }^{t} k_{2 n}^{s}\right]-\left({ }^{t} k_{12}^{b}{ }^{t} k_{21}^{b}\right)_{n}
\end{aligned}
$$

Where left superscript ' $t$ ' represents tangent quantity; $K_{i j}^{b}$ are the relevant stiffness coefficients of stiffness matrix; $K_{\text {in }}^{s}$ are the stiffness of section springs at two ends of element. In computational practice the stiffness of section springs $K^{s}{ }_{i n}$ can be assigned a very large or a very small stiffness according to whether the plastic hinge comes into being. This section spring model can be extended to refined plastic hinge model where the stiffness of the spring is varied from infinity to zero and in this situation the tangent and secant spring stiffness can be different.

\section{FORCE RECOVERY PROCEDURE IN PLASTIC HINGE ANALYSIS}

Before the corrector or force recovery procedure is exercised in the incremental iterative analysis, the element deformation ${ }^{0}[u]$ in last configuration and the present element deformation increments $[\Delta u]$ have been extracted from the system incremental displacements which are computed by the predictor equations. The task of the force recovery procedure is to recover the element internal forces $[f]$ at the current configuration.

\subsection{Total Secant Iterative Method}

The element internal forces can be calculated directly by the secant stiffness in Eqs. 1 to 4 with the present element deformation $[u]$ cumulated from the element deformation ${ }^{0}[u]$ in last configuration and the present deformation increments $[\Delta u]$. The difficulty arises due to the fact that the expression for member axial force as given by Eq. 3 involves bowing functions which in turn are functions of the axial force parameter $q=P L^{2} / E I$. As a result the whole equations are implicit functions of axial force $P$. To solve this problem, an axial force iteration procedure has to be adopted which is based on first-order Taylor series expansion of Eq. 3.

As the secant stiffness equations are explicit and exact, this force recovery method has the capability of good and rapid convergence than the force incremental method in elastic range. If plastic hinge behavior is considered, there is abrupt point in the element force-deformation curve when plastic hinge comes into being. The explicit secant stiffness relationship does exist in simple situation but has different forms for elastic and plastic range. The situation is further complicated as axial force iteration procedure must be adopted to obtain the exact axial force value which is the basis of determining the exact position of yield surface. So this force recovery method is recommended only for the elastic nonlinear analysis of frames.

\subsection{Force Incremental Method}

The element recovery forces can also be cumulated from the force increments which can be calculated by the product of element natural stiffness and natural deformations

$$
\Delta f=k_{e} \Delta u
$$


in which $k_{\mathrm{e}}$ is the element natural stiffness matrix. The internal forces of the element can be updated by adding the incremental element forces to the element internal forces in last configuration

$$
f={ }^{0} f+\Delta f
$$

The accuracy of force increments is only approached in the first order of the displacement increments so the load increment step should be small enough to obtain a sufficiently accurate solution.

The secant iterative method has obstacle to cope with the abrupt point in plastic hinge analysis. In incremental type force recovery methods things get simple and natural: firstly scale back to the right position where plastic hinge occurs, then calculate the force increment causing by deformation increment remained using the modified incremental stiffness. The whole procedure can be illustrated as follows

$$
\begin{aligned}
& { }^{e} f=f-\alpha \Delta f \\
& \Delta f^{\prime}=k_{e}^{\prime} \Delta u \\
& { }^{p} f={ }^{e} f+\alpha \Delta f^{\prime} \\
& \alpha=\frac{M-M_{p}}{\Delta M}
\end{aligned}
$$

Where ${ }^{e} f$ and ${ }^{p} f$ are element internal forces in elastic and plastic range respectively; $k_{e}$ ' is the modified incremental stiffness after plastic hinge formed and $\alpha$ is a scale factor that reverse the force to the right position where plastic hinge occurs. When the moment axial force interaction equations such as Eqs. 6-7 are introduced as yield surface, a one dimensional search procedure should be adopted to find the exact scale factor instead of Eq. 25 being used directly.

Actually the force-deformation relationship is not a straight line but a curve with abrupt point as illustrated by Figure 1. Because of the tangent approximation, the force increment method can only get force-deformation path $\mathrm{OA}^{\prime} \mathrm{B}^{\prime}$, which has the equal internal forces with the real path OAB but the deformation where plastic hinge occurs is different. This is not a problem as the load cycle step should be small enough in force incremental method and the introduced error can be neglected.

\subsection{Total Incremental Iterative Method}

The secant iterative method is more accurate and rapid while the force incremental method has the advantage of simplicity and flexibility of coping with abrupt point on force-deformation curve. Their combined use is a sensible choice in improving the computational accuracy and efficiency, just like the incremental-iterative method used in the solution of system equilibrium equations. Simulating the modified Newton-Raphson method, the total incremental iteration can be written as

$$
\begin{gathered}
{ }^{i}[\Delta f]={ }^{i}[k][\Delta u] \\
{ }^{i}[f]={ }^{i-1}[f]+{ }^{i}[\Delta f] \\
{ }^{i}[u]={ }^{i-1}[u]+{ }^{i}[\Delta u]
\end{gathered}
$$




$$
{ }^{i+1}[\Delta u]={ }^{0}[u]+\sum_{k=1}^{i}{ }^{k}[\Delta u]-{ }^{i}[u]
$$

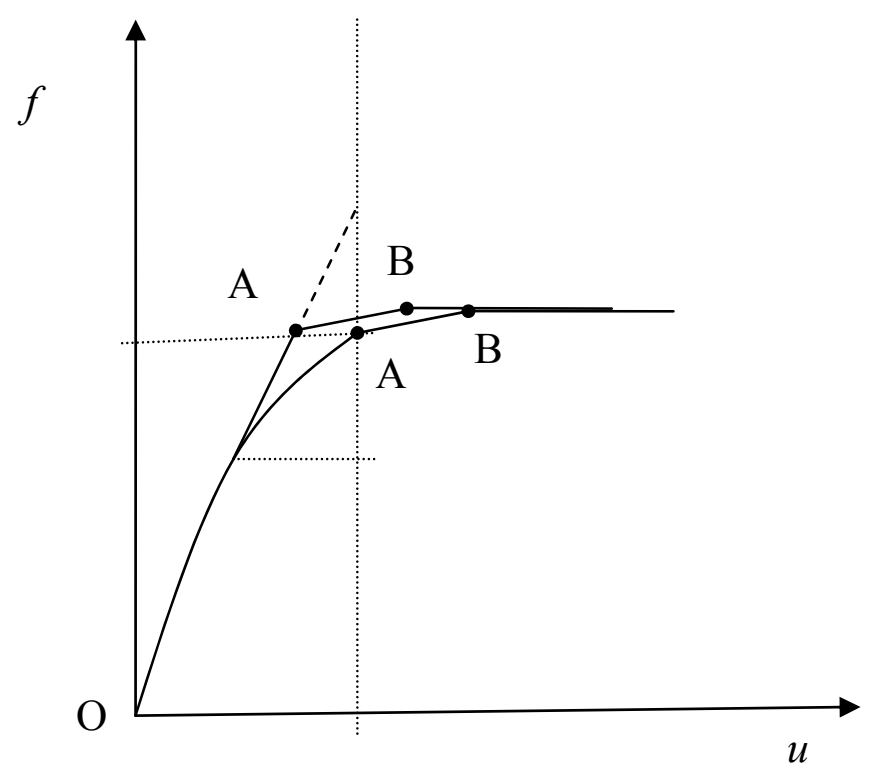

Figure 1. Illustration of Different Force Recovery Method

In this iteration the total deformation ${ }^{\mathrm{i}}[u]$ can be easily calculated by the reverse form of secant stiffness Eqs. 1-4. This iterative process of Eqs. 26-29 continues until the unbalanced deformation ${ }^{\mathrm{i}}[\Delta u]$ is sufficiently small. And it should be noted that the tangent matrix in Eq. 26 is kept unchanged during the iteration, which makes this iteration use minimal computational effort compared with system equation solution.

The same as the force incremental method, a one dimensional search procedure should be adopted to scale member state right back to the yield surface and the exact scale factor can be obtained. Unlike the force incremental method, the exact point A that plastic hinge occurs can be converged by the iteration procedure. If the explicit secant stiffness relationships with plastic hinge exist, another iteration as Eqs. 26-29 can be continued to arrive the specified deformation state. Otherwise Eq. 24 can be utilized in the case of only incremental force-deformation can be obtained conveniently when refined plastic hinge model is used. As the force recovery method can simulate the member exactly in the existence of plastic hinge, especial load increment sizes control technique to scale the whole structure back to plastic hinge point is not needed.

\section{NUMERICAL EXAMPLES}

\subsection{Vogel Portal Frame}

Figure 2 shows a portal frame proposed and solved by Vogel. The frame possesses an out-of-plumbness of 1/400 and a member initial imperfection of 1/1000 along the column length. The section of the beam is HEA-340 and that of columns is HEB-300. This paper uses a single element and elastic plastic hinge model with the proposed force recovery method to model the beam and the column. The computed ultimate load factor is 1.00 which is close to the result of [17]. Load-displacement curves by different methods are showed in Figure 3. The total incremental iterative method and force incremental method give almost same results, but the total incremental 
iterative method can still obtain approximate collapse load even with 10 load cycles. It is viewed from numerical iteration process that the total incremental iterative method has nearly same performance in elastic and plastic range, with 2 or 3 more iterations increasing in presence of plastic hinge.

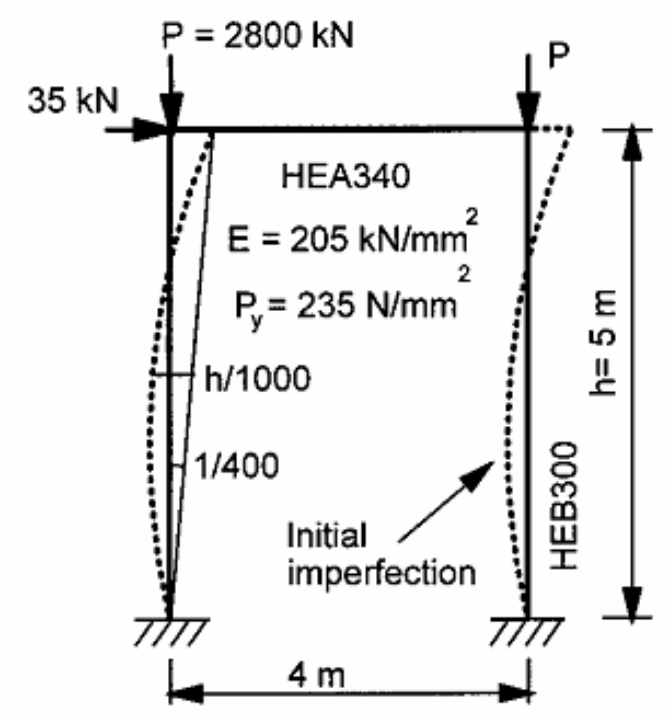

Figure 2. Vogel Portal Frame

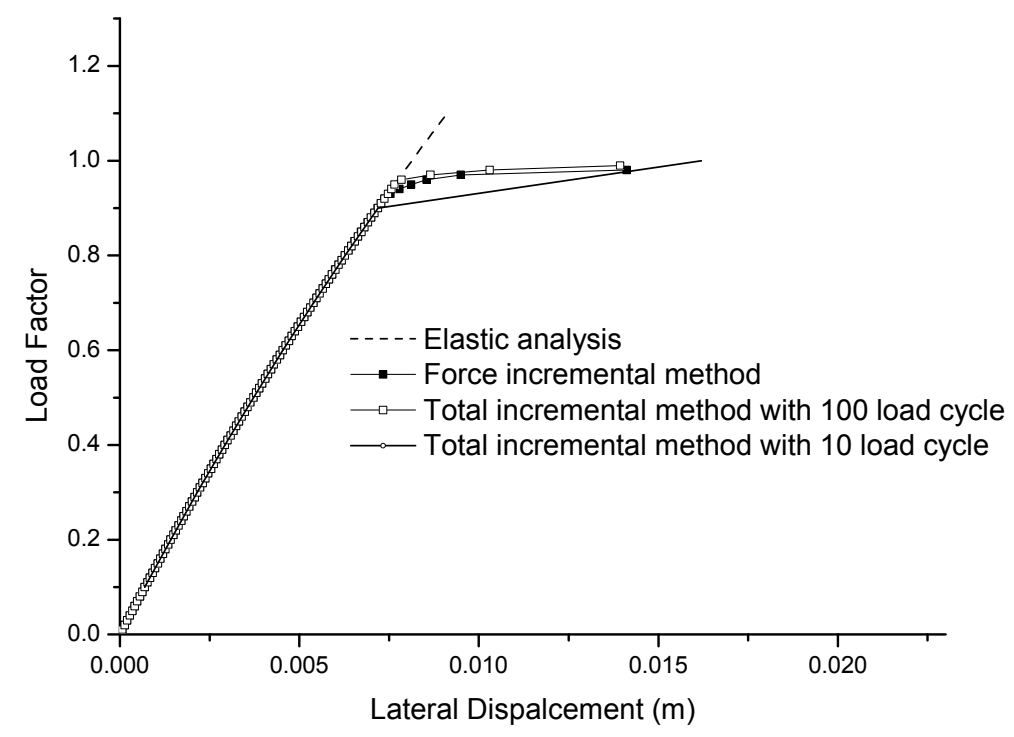

Figure 3. Load-displacement Curve of Vogel Portal Frame

\subsection{Vogel Six Story Frame}

Figure 4 shows the well known Vogel six story frame. The frame possesses an out-of-plumbness of $1 / 400$ and a member initial imperfection of $1 / 1000$ along the column length. The section of the beam is IPE-240 to IPE-400 and that of columns is HEB-200 to HEB-260. This paper uses a single element and elastic plastic hinge model with the proposed force recovery method to model the beam and the column. The computed ultimate load factor is 1.1 which is which is close to the result of [17]. From Figure 5 it can be seen that almost same collapse load is obtained by the total incremental iterative method with different load steps. As the exact load path is not always needed in design, the proposed method is effective and rapid, which is demanded and welcomed by engineers. Figure 6 shows the bending moment diagram and plastic hinge position of Vogel six story frame near collapse. 


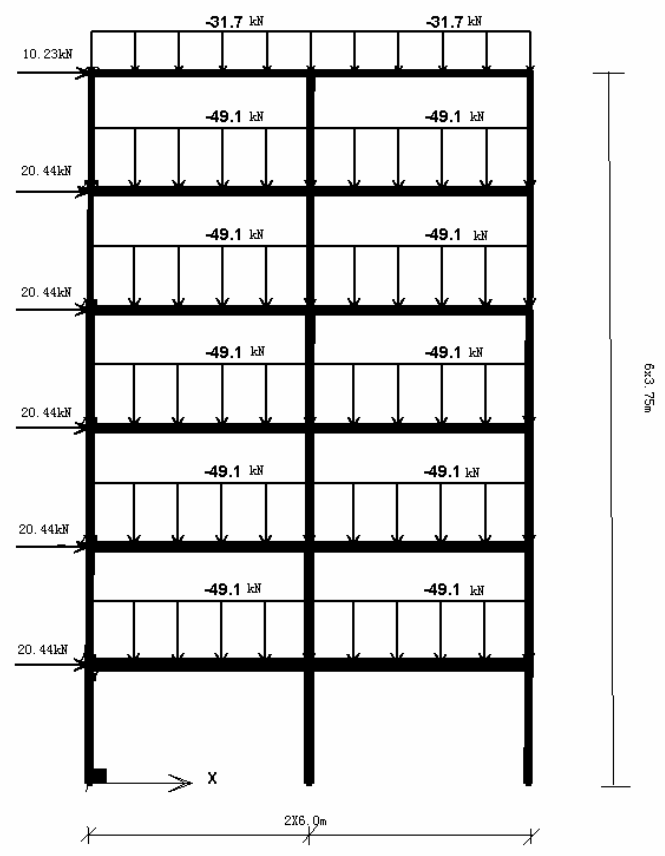

Figure 4. Vogel Six Story frame

\section{CONCLUSION}

This paper provides a detailed description about the application of the total incremental iterative force recovery method in the plastic hinge analysis of framed structures. This method utilizes both the secant and tangent stiffness and remains incremental manner in the process of recovering internal forces, which is convenient to the modeling of plastic hinge. As the force recovery method can simulate the member exactly in the existence of plastic hinge, especial load increment sizes control technique to scale the whole structure back to plastic hinge point is not needed. Numerical examples show this method is useful in the plastic hinge analysis and daily design of steel frames.

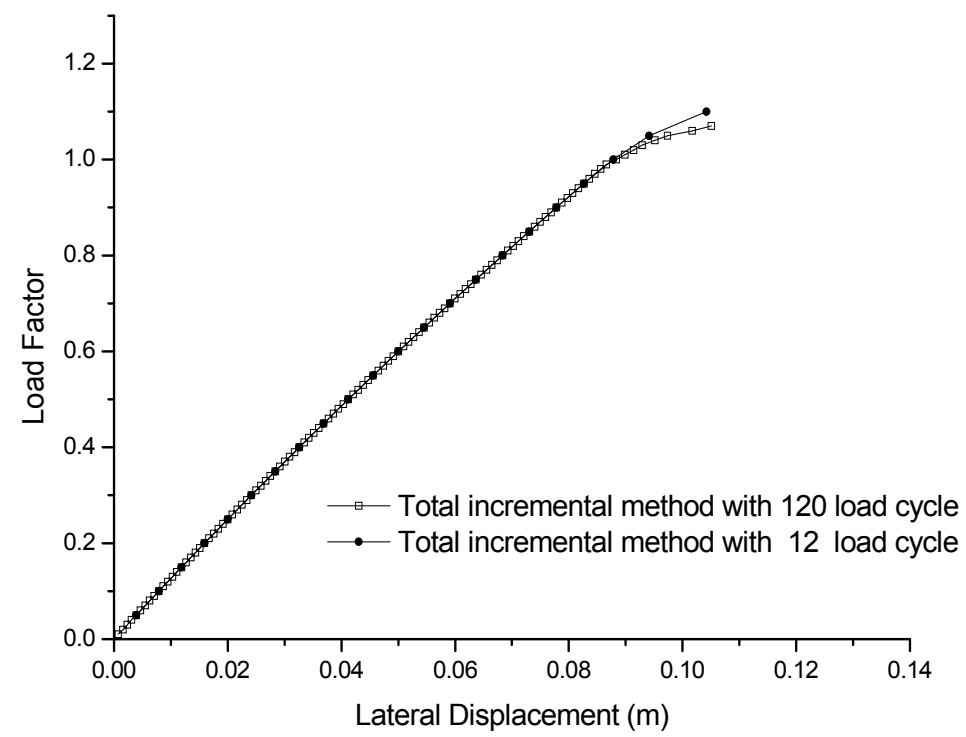

Figure 5. Load-displacement Curve of Vogel Six Story Frame 


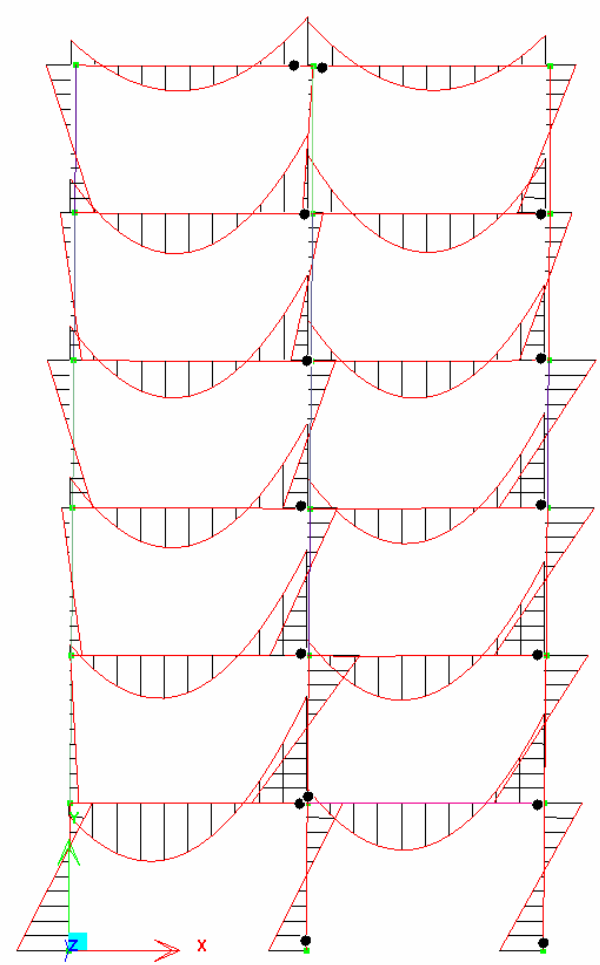

Figure 6. Bending Moment Diagram and Plastic Hinge Position of Vogel Six Story Frame

\section{REFERENCES}

[1] Wang, F.W. and Liu, Y.P., "Total and Incremental Iteration Force Recovery Procedure for the Nonlinear Analysis of Framed Structures", Advanced Steel Construction, 2009, Vol. 5, pp.500-514.

[2] Liew, J.Y.R., White, D.W. and Chen, W.F., "Second-order Refined Plastic-hinge Analysis for Frame Design. Part I", Journal of Structural Engineering, ASCE, 1993, Vol. 119, pp. 3196-3216.

[3] Liew, J.Y.R., White, D.W. and Chen W.F., "Second-order Refined Plastic-hinge Analysis for Frame Design. Part II", Journal of Structural Engineering, ASCE, 1993, Vol. 119, pp. 3217-3237.

[4] Zhou, Z.H. and Chan, S.L., "Elastoplastic and Large Deflection Analysis of Steel Frames by One Element Per Member. I: One Hinge Along Member", Journal of Structural Engineering, ASCE, 2004, Vol. 130, pp. 538-544.

[5] Chan, S.L. and Zhou, Z.H., "Elastoplastic and Large Deflection Analysis of Steel Frames by One Element Per Member. II: Three Hinges Along Member", Journal of Structural Engineering, ASCE, 2004, Vol. 130, pp. 545-553.

[6] Yang, Y.B., Lin, S.P. and Leu, L.J., "Solution Strategy and Rigid Element for Nonlinear Analysis of Elastically Structures Based on Updated Lagrangian formulation", Engineering Structures, 2007, Vol. 29, pp. 1189-1200.

[7] Chan, S.L. and Chui, P.P.T., "Non-linear Static and Cyclic Analysis of Steel Frames with Semi-rigid Connections", Elsevier Science Ltd., 2000.

[8] Chen, H., "Nonlinear Inelastic Analysis of Steel-concrete Composite Frames." PhD thesis, Dept. of Civil Engineering, National Univ. of Singapore, Singapore, 2000.

[9] Liew, J. Y. R., Chen, H., Shanmugam, N.E. and Chen, W.F., "Improved Nonlinear Plastic Hinge Analysis of Space Frame Structures", Engineering Structures, 2000, Vol. 22, No.. 10, pp. 1324-1338. 
[10] Liew, J.Y.R., and Tang, L.K., "Advanced Plastic Hinge Analysis For the Design of Tubular Space Frames”, Engineering Structures, 2000, Vol. 22, No. 7, pp. 769-783.

[11] Wang, F.W. and Chan, S.L., "Optimization and Sensitivity Analysis of Space Frames Allowing for Large Deflection”, Engineering Structures, 2006, Vol. 28, pp. 1395-1406.

[12] Chan, S.L. and Kitipornchai, S., "Geometric Nonlinear Analysis of Asymmetric Thin-walled Beam-columns", Engineering Structures, 1987, Vol.9, pp. 243-254.

[13] Gu, J.X., "Large Displacement Elastic Analysis of Space Frames Allowing for Flexural-torsional Buckling of Beams", Dept. of Civil and Structural Engineering, Hong Kong Polytechnic University, Hong Kong, 2004.

[14] Chan, S.L. and Zhou, Z.H., "Pointwise Equilibrating Polynomial Element for Nonlinear Analysis of Frames", Journal of Structural Engineering, 1994, Vol. 120, No. 6, pp. 1703-1717.

[15] Iu, C.K. and Chan, S.L., "A Simulation-based Large Deflection and Inelastic Analysis of Steel Frames under Fire". Journal of Constructional Steel Research, 2004, Vol. 60, pp. 1495-524.

[16] Iu, C.K., "Inelastic Finite Element Analysis of Composite Beam on the Basis of Plastic Hinge Approach", Engineering Structures, 2008, Vol. 30, pp. 291-302.

[17] Toma, S. and Chen, W.F., "European Calibration Frames for Second-order Inelastic Analysis", Engineering Structures, 1992, Vol. 14, No. 1, pp. 7-14. 